\title{
Emergence of extensively drug-resistant Acinetobacter baumannii complex over 10 years: Nationwide data from the Taiwan Surveillance of Antimicrobial Resistance (TSAR) program
}

\author{
Shu-Chen Kuo 1,2,3, Shan-Chwen Chang ${ }^{4,5}$, Hui-Ying Wang ${ }^{1}$, Jui-Fen Lai', Pei-Chen Chen'1 Y Yih-Ru Shiau',
} I-Wen Huang ${ }^{1}$ and Tsai-Ling Yang Lauderdale ${ }^{1 *} ;$ TSAR Hospitals

\begin{abstract}
Background: Acinetobacter baumannii complex ( $A B C$ ) has emerged as an important pathogen causing a variety of infections. Longitudinal multicenter surveillance data on ABC from different sources in Taiwan have not been published. Using data from the Taiwan Surveillance of Antimicrobial Resistance (TSAR) conducted biennially, we investigated the secular change in resistance of 1640 ABC from 2002 to 2010 (TSAR period III to VII) to different antimicrobial agents and identified factors associated with imipenem-resistant and extensively drug-resistant $A B C$ (IRABC and XDRABC).

Methods: Isolates were collected by TSAR from the same 26 hospitals located in all 4 regions of Taiwan. Minimum inhibitory concentrations (MIC) were determined by reference broth microdilution method. Isolates nonsusceptible to all tested aminoglycosides, fluoroquinolones, $\beta$-lactam, $\beta$-lactam/ $\beta$-lactam inhibitors, and carbapenems were defined as extensively drug-resistant (XDR). Multivariate logistic regression analysis was performed to assess the relationship between predictor variables among patients with resistant $A B C$ and patients with non-resistant $A B C$.

Results: The prevalence of IRABC increased from 3.4\% in 2002 to $58.7 \%$ in 2010 ( $P<0.001$; odds ratio [OR], 2.138; $95 \%$ confidence interval [CI], 1.947 to 2.347) and that of XDRABC increased from $1.3 \%$ in 2002 to $41.0 \%$ in 2010 $(P<0.001 ; \mathrm{OR}, 1.970 ; 95 \% \mathrm{Cl}, 1.773-2.189)$. The rates of non-susceptibility to other antimicrobial agents remained high ( $>55 \%)$ over the years with some fluctuations before and after TSAR V (2006) on some agents. Multivariate analysis revealed that recovery from elderly patients, origins other than blood, from ICU settings, or geographic regions are independent factors associated with IRABC and XDRABC. Although the prevalence of XDRABC increased in all four regions of Taiwan over the years, central Taiwan had higher prevalence of XDRABC starting in 2008. Susceptibility to polymyxin remained high (99.8\%).

Conclusions: This longitudinal multicenter surveillance program revealed significant increase and nationwide emergence of IRABC and XDRABC in Taiwan over the years. This study also identified factors associated with IRABC and XDRABC to help guide empirical therapy and at-risk groups requiring more intense interventional infection control measures with focused surveillance efforts.
\end{abstract}

Keywords: Extensively drug-resistant, Acinetobacter baumannii complex, Antimicrobial resistance

\footnotetext{
* Correspondence: lauderdale@nhri.org.tw

${ }^{1}$ National Institute of Infectious Diseases and Vaccinology, National Health Research Institutes, No. 35 Keyan Road, Zhunan, Taiwan 35053

Full list of author information is available at the end of the article
} 


\section{Background}

Acinetobacter spp., especially Acinetobacter baumannii complex $(\mathrm{ABC})$, has emerged as an important pathogen causing a variety of infections including urinary tract infection, skin and soft tissue infections, and pneumonia and bloodstream infections with high morbidity and mortality [1]. The ability to chronically colonize patients and cause outbreaks which are usually hard to eradicate poses significant challenge to infection control and increases healthcare expenditure [2]. In addition to its intrinsic resistance to many commonly used antibiotics, this troublesome pathogen can gain additional mechanism rapidly in response to new broad-spectrum antibiotics $[3,4]$. Due to treatment failure, drug-resistant strains have been associated with higher mortality and prolonged hospital stay compared with susceptible ones $[5,6]$.

Carbapenems such as imipenem and meropenem are the last resort of drugs for the treatment of multidrugresistant pathogens including $\mathrm{ABC}$. However, the incidence of carbapenem resistance in $\mathrm{ABC}$ increased steadily in the 2000s [4,7]. In Europe, the MYSTIC program in 2006 revealed a considerable increase in carbapenem resistance rates to $42.5 \%$ [8]. Worldwide, the SENTRY program documented an overall increase in imipenem nonsusceptibility from $34.5 \%$ in 2006 to $59.8 \%$ in 2009 [9]. Imipenem-resistance in Taiwan ranged from $22 \%$ in 2000 to $25 \%$ in 2005 [10]. Ampicillin/sulbactam, tigecycline, and colistin are possible options for imipenem-resistant $\mathrm{ABC}$ but decreasing susceptibility to these agents has also been reported [1]. Surveillance is therefore important in providing useful information for physicians in choosing empirical antibiotics. It also helps to address specific resistant issues within a region to help identify targeted intervention measures [11,12].

Although there have been reports of the high prevalence of drug-resistant $\mathrm{ABC}$ in Taiwan [13,14], longitudinal nationwide surveillance data on isolates from different sources in Taiwan have not been published. The Taiwan Surveillance of Antimicrobial Resistance (TSAR) is a nationwide program at the National Health Research Institutes [11] and has been conducted biennially since 1998 [15]. Using data from TSAR, we aimed at detailing the secular change of resistance to various antimicrobial agents in $\mathrm{ABC}$ from different sources over 10 years and identify factors associated with imipenem-resistant and extensively drug-resistant ABC (IRABC and XDRABC).

\section{Methods}

\section{Study period and isolate collection process}

The study period spanned from 2002 to 2010 (corresponding to TSAR period III to VII). Bacterial isolates were collected biennially from July to September by the TSAR program from the same 26 hospitals except TSAR
V (2006), in which one hospital did not participate. These hospitals comprised 11 medical centers and 15 regional hospitals, and are located in all 4 regions of Taiwan including $7,8,8$, and 3 in the north, central, south and east region, respectively. The majority of the Taiwan's population lives in the western part (north, central and south regions) while the eastern part is the least populated region. The collection protocol was similar for all 5 rounds of TSAR as described previously [16,17]. Briefly, each hospital first collected 50 outpatient isolates, 30 adult ICU and 100 non-ICU inpatient isolates, and 20 pediatric isolates. After completion of the above collection, an additional 20 (for TSAR III to V) to 50 (for TSAR VI and VII) isolates from blood and sterile body sites were collected. The isolates were collected sequentially without specifying species. All isolates were stored at $-80^{\circ} \mathrm{C}$ for subsequent testing. The bacterial isolates were recovered from clinical samples taken as part of standard care. The study was approved by the Research Ethics Committee of National Health Research Institutes (EC960205).

\section{Bacterial isolates and information}

For Acinetobacter spp., isolates were subcultured to blood agar and McConkey agar plates at our laboratory for purity check and to confirm species identification. Either Vitek I (prior to 2008) or Vitek II (2008 and 2010) GN card was used (bioMérieux, Marcy l'Etoile, France). In addition, conventional biochemical tests including oxidase, Triple Sugar Iron, $42^{\circ} \mathrm{C}$, malonate, and hemolysis on sheep blood agar were used to aid in confirmation of the A. baumannii complex [18]. The hospital also provided information on specimen source and patient age. For analysis, samples from upper respiratory tract were designated as respiratory origin. Samples from pus/discharge included those from abscesses or wounds.

\section{Antimicrobial susceptibility}

Minimum inhibitory concentrations (MIC) of different agents were determined by reference broth microdilution test following the guidelines of Clinical and Laboratory Standards Institute (CLSI) using custom designed Sensititre panels (Trek Diagnostics, West Essex, England) [19] Amikacin, ampicillin/sulbactam, ceftazidime, cefepime, ciprofloxacin, gentamicin, levofloxacin, imipenem, and piperacillin/tazobactam were tested on all isolates from each study year. Polymyxin B and tigecycline were tested on imipenem-resistant isolates in 2002 and 2004 and on all isolates from 2008-2010. Trimethoprim-sulfamethoxazole was tested on all isolates from 2002, 2004 and 2010. Control strains included Pseudomonas aeruginosa ATCC 27853, Escherichia coli ATCC 25922 and ATCC 35218. Results were interpreted using the CLSI breakpoints except for colistin 
and tigecycline, for which the EUCAST breakpoints (for Enterobacteriaceae) were used (http://www.eucast.org/ clinical_breakpoints). Isolates nonsusceptible to all the tested aminoglycosides, fluoroquinolones, $\beta$-lactam, $\beta$ lactam/ $\beta$-lactam inhibitors, and carbapenems were defined as extensively drug-resistant (XDR) [20,21].This is a modification of the ECDC definition for XDR, which is defined as non-susceptibility to at least one agent in all but two or fewer antimicrobial categories [20].

\section{Data and statistical analysis}

For analysis of susceptibility rates in different year and patient groups, we used the Whonet software [22]. Univariate analysis was done using Student's $t$ test/MannWhitney U test, or Fisher's exact tests as appropriate. Multivariate logistic regression analysis was performed to assess the relationship between predictor variables among patients with resistant $\mathrm{ABC}$ and patients with non-resistant $\mathrm{ABC}$. The variables included those identified in the univariate analysis as possibly being associated with resistance rate $(P<0.05)$. For comparison of resistance between regions and specimen types, these variables were entered in the multivariate analysis in a dummy form (resistance rate in northern Taiwan or that of blood samples as the reference group, respectively). For the trend test calculation, a continuous variable
(TSAR period III to VII corresponding to 3 to 7, respectively) was used [23]. All analyses were performed with the Statistical Package for the Social Sciences version 18.0 (SPSS, Chicago, IL, USA). A $P<0.05$ was considered to be statistically significant.

\section{Results}

\section{Bacterial isolates}

Between 2002 and 2010, a total of 1,681 Acinetobacter spp. isolates were collected and $\mathrm{ABC}$ comprised $97.6 \%$ (1,640 isolates). Non-ABC isolates were excluded from subsequent data analysis. Among the $1640 \mathrm{ABC}$ isolates, the mean age of patients was $66.8 \pm 19.8$ years. Table 1 lists the source breakdown of the isolates for each round of TSAR. Isolates were mostly recovered from respiratory samples (822 isolates, 50.1\%), followed by blood $(244,14.9 \%)$, pus/discharge $(219,13.4 \%)$ and urine $(216$, $13.2 \%)$. Seven hundred and thirty-five $(44.8 \%)$ were from medical centers and 600 (36.6\%) were from ICU. Isolates from central Taiwan comprised the largest proportion (627, 38.2\%) (Table 1).

\section{Changes in non-susceptibility to different antimicrobial agents over the years}

Rates of non-susceptibility to different antimicrobial agents between 2002 and 2010 are shown in Table 2.

Table 1 Source breakdown of Acinetobacter baumannii complex from the Taiwan Surveillance of Antimicrobial Resistance (TSAR) by year

\begin{tabular}{|c|c|c|c|c|c|c|c|c|c|c|c|c|c|}
\hline \multirow[t]{2}{*}{ Strata } & \multicolumn{2}{|c|}{ TSAR III (2002) } & \multicolumn{2}{|c|}{ TSAR IV (2004) } & \multicolumn{2}{|c|}{ TSAR V (2006) } & \multicolumn{2}{|c|}{ TSAR VI (2008) } & \multicolumn{2}{|c|}{ TSAR VII (2010) } & \multicolumn{2}{|c|}{ TSAR III-VII Combined } & \multirow[t]{2}{*}{$P^{a}$} \\
\hline & $\mathbf{N}$ & $\%$ & $\mathbf{N}$ & $\%$ & $\mathbf{N}$ & $\%$ & $\mathbf{N}$ & $\%$ & $\mathbf{N}$ & $\%$ & $\mathbf{N}$ & $\%$ & \\
\hline \multicolumn{14}{|l|}{ Hospital type } \\
\hline Medical centers (11) & 162 & 54.4 & 124 & 47.9 & 126 & 41.4 & 172 & 41.1 & 151 & 41.8 & 735 & 44.8 & 0.002 \\
\hline Regional hospitals (15) & 136 & 45.6 & 135 & 52.1 & 178 & 58.6 & 246 & 58.9 & 210 & 58.2 & 905 & 55.2 & 0.002 \\
\hline \multicolumn{14}{|l|}{ Region } \\
\hline North (7) & 90 & 30.2 & 70 & 27.0 & 76 & 25.0 & 118 & 28.2 & 118 & 32.7 & 472 & 28.8 & 0.2354 \\
\hline Central (8) & 94 & 31.5 & 91 & 35.1 & 109 & 35.9 & 189 & 45.2 & 144 & 39.9 & 627 & 38.2 & 0.0024 \\
\hline South (8) & 77 & 25.8 & 57 & 22.0 & 76 & 25.0 & 65 & 15.6 & 68 & 18.8 & 343 & 20.9 & 0.0032 \\
\hline East (3) & 37 & 12.4 & 41 & 15.8 & 43 & 14.1 & 46 & 11.0 & 31 & 8.6 & 198 & 12.1 & 0.0194 \\
\hline \multicolumn{14}{|l|}{ Patient location } \\
\hline $\mathrm{ICU}$ & 100 & 33.6 & 105 & 40.5 & 103 & 33.9 & 157 & 37.6 & 135 & 37.4 & 600 & 36.6 & 0.3848 \\
\hline Non-ICU & 174 & 58.4 & 138 & 53.3 & 165 & 54.3 & 227 & 54.3 & 201 & 55.7 & 905 & 55.2 & 0.7578 \\
\hline OPD/ER & 24 & 8.1 & 16 & 6.2 & 36 & 11.8 & 34 & 8.1 & 25 & 6.9 & 135 & 8.2 & 0.1104 \\
\hline \multicolumn{14}{|l|}{ Specimen type } \\
\hline Respiratory & 157 & 52.7 & 136 & 52.5 & 137 & 45.1 & 208 & 49.8 & 184 & 51.0 & 822 & 50.1 & 0.3294 \\
\hline Blood & 33 & 11.1 & 28 & 10.8 & 34 & 11.2 & 84 & 20.1 & 65 & 18.0 & 244 & 14.9 & 0.0002 \\
\hline Pus/discharge & 49 & 16.4 & 40 & 15.4 & 56 & 18.4 & 40 & 9.6 & 34 & 9.4 & 219 & 13.4 & 0.0005 \\
\hline Urine & 34 & 11.4 & 30 & 11.6 & 50 & 16.4 & 57 & 13.6 & 45 & 12.5 & 216 & 13.2 & 0.332 \\
\hline Others & 25 & 8.4 & 25 & 9.7 & 27 & 8.9 & 29 & 6.9 & 33 & 9.1 & 139 & 8.5 & 0.7321 \\
\hline Total & \multicolumn{2}{|c|}{298} & \multicolumn{2}{|c|}{259} & \multicolumn{2}{|c|}{304} & \multicolumn{2}{|c|}{418} & \multicolumn{2}{|c|}{361} & \multicolumn{2}{|c|}{1640} & \\
\hline
\end{tabular}

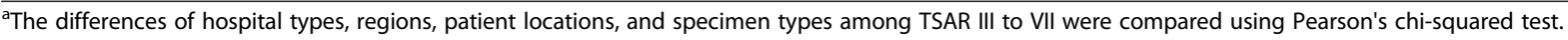


The secular trend of non-susceptibility to amikacin, ceftazidime, levofloxacin, and imipenem over the 10 years is also shown in Figure 1 to highlight the sharp increase of carbapenem (imipenem) non-susceptibility from $3.4 \%$ in 2002 to $58.7 \%$ in $2010(P<0.001$; odds ratio [OR], 2.138; $95 \%$ confidence interval [CI], 1.947 to 2.347 ). The increased carbapenem-resistance was observed in isolates from elderly as well as non-elderly patients, and in those from different specimen types, ICU and non-ICU patients, and throughout different regions of Taiwan (Figure 2).

The rates of non-susceptibility to other antimicrobial agents remained high $(>55 \%)$ over the years although fluctuations were observed before and after TSAR V (2006) on some agents (Table 2 \& Figure 1); non-susceptibility to amikacin, cefepime, ceftazidime, levofloxacin, and piperacillin/tazobactam increased significantly before 2006 (TSAR V), then stayed at a plateau and even declined thereafter (Table 2). In contrast, the rates of nonsusceptibility to ampicillin/sulbactam (range 56.1\% 64.1\%) fluctuated over 10 years without significant change ( $P=0.775$; OR, 1.010; 95\% CI, 0.942 to 1.084$)$, while that of gentamicin decreased slightly since 2004 ( $P=0.034$; OR, 0.883 ; $95 \%$ CI, 0.787 to 0.991$)$. The overall rate of susceptibility to tigecycline $(\mathrm{MIC}<=2 \mathrm{mg} / \mathrm{L})$ and polymyxin $\mathrm{B}$ was $97.7 \%$ and $99.8 \%$, respectively in 1,160 tested isolates and $98.1 \%$ and $100 \%$, respectively in IRABC.

Factors associated with emergence of imipenem-resistant A. baumannii complex (IRABC)

Table 3 presents the factors associated with the emergence of IRABC. Isolates from elderly patients ( $>65$ years old), respiratory tract origin, ICU settings, or central
Taiwan were significantly associated with imipenem resistance whereas rates of imipenem-resistance were lower in isolates from blood or pus/discharge, medical centers, southern or northern Taiwan (Figure 2). Multivariate analysis revealed recovery from elderly patients, origins other than blood, ICU settings, geographic region, and latter collection year remained independent factors (Table 3).

\section{Emergence of extensively drug-resistant $A$. baumannii complex (XDRABC)}

The prevalence of XDRABC increased significantly from $1.3 \%$ in 2002 to $41.0 \%$ in 2010 , respectively $(P<0.001$; OR, 1.970; 95\% CI, 1.773-2.189), with an overall prevalence of $26.1 \%$ (428 isolates) over the 10 years. The increased XDRABC also occurred in isolates from elderly and non-elderly patients both, and in those from different specimen types, ICU as well as non-ICU patients, while central region saw a sharp increase of XDRABC in 2008-2010 (Figure 3). Ten (2.3\%) of the XDRABC had tigecycline $>2 \mathrm{mg} / \mathrm{L}$ and all were susceptible to polymyxin $B$. Trimethoprim-sulfamethoxazole was tested on 187 XDRABC isolates and all but 4 were resistant.

\section{Factors associated with emergence of XDRABC}

Factors associated with XDRABC strains included recovery from elderly patients (> 65 years old), respiratory tract origin, ICU settings, or central Taiwan (Table 4). The extensively drug-resistant rates over 10 years stratified by these independent factors are shown in Figure 3. Isolates from blood or pus/discharge, medical center, southern or northern Taiwan were less likely to be XDR.

Table 2 Secular trend of antimicrobial non-susceptibility in Acinetobacter baumannii complex from the 2002 to 2010 Taiwan Surveillance of Antimicrobial Resistance (TSAR) program

\begin{tabular}{|c|c|c|c|c|c|c|c|c|c|c|c|}
\hline \multirow{2}{*}{$\begin{array}{l}\text { Antimicrobial } \\
\text { agents }^{\mathrm{a}}\end{array}$} & \multicolumn{5}{|c|}{ Resistance rate (\%) by year } & \multicolumn{3}{|c|}{2002 to $2006^{b}$} & \multicolumn{3}{|c|}{2006 to 2010} \\
\hline & $2002(298)$ & 2004 (259) & $2006(304)$ & $2008(418)$ & $2010(361)$ & $P$ & OR & $95 \% \mathrm{Cl}$ & $P$ & OR & $95 \% \mathrm{Cl}$ \\
\hline Amikacin & 63.1 & 68 & 70.7 & 66.5 & 64.3 & 0.046 & 1.19 & $1.003-1.411$ & 0.081 & 0.865 & $0.735-1.018$ \\
\hline Amp/Sulb & 57.4 & 64.1 & 57.6 & 61.5 & 59.6 & 0.971 & 1.003 & $0.852-1.180$ & 0.637 & 1.038 & $0.889-1.212$ \\
\hline Cefepime & 64.1 & 70.7 & 74 & 71.3 & 60.9 & 0.008 & 1.265 & $1.062-1.507$ & $<0.001$ & 0.731 & $0.619-0.864$ \\
\hline Ceftazidime & 68.1 & 73.4 & 76.6 & 74.6 & 70.4 & 0.019 & 1.241 & $1.036-1.486$ & 0.063 & 0.848 & 0.712-1.009 \\
\hline Ciprofloxacin & 68.8 & 75.3 & 76 & 75.4 & 75.3 & 0.047 & 1.202 & $1.002-1.441$ & 0.853 & 0.983 & $0.824-1.174$ \\
\hline Gentamicin & 73.5 & 78.8 & 76.6 & 70.3 & 72.9 & 0.367 & 1.09 & 0.904-1.315 & 0.311 & 0.915 & 0.770-1.087 \\
\hline Imipenem & 3.4 & 18.1 & 31.6 & 51.4 & 58.7 & $<0.001$ & 3.043 & $2.348-3.944$ & $<0.001$ & 1.732 & $1.478-2.029$ \\
\hline Levofloxacin & 66.1 & 72.6 & 74.3 & 73.9 & 71.2 & 0.027 & 1.222 & $1.023-1.458$ & 0.349 & 0.921 & 0.775-1.094 \\
\hline Pip/Tazo & 62.8 & 76.8 & 75.3 & 74.6 & 68.4 & 0.001 & 1.363 & $1.139-1.631$ & 0.041 & 0.835 & 0.703-0.992 \\
\hline TMP/SMX & 73.8 & 75.7 & $N T^{c}$ & NT & 71.5 & $N D^{d}$ & & & ND & & \\
\hline
\end{tabular}

${ }^{a}$ Amp/Sulb, Ampicillin/sulbactam; Pip/Tazo, Piperacillin/tazobactam; TMP/SMX, trimethoprim/sulfamethoxazole.

${ }^{\mathrm{b}} \mathrm{OR}$, odds ratio; $\mathrm{Cl}$, confidence interval.

${ }^{\mathrm{c}} \mathrm{NT}$, not tested.

${ }^{\mathrm{d}} \mathrm{ND}$, not done. 


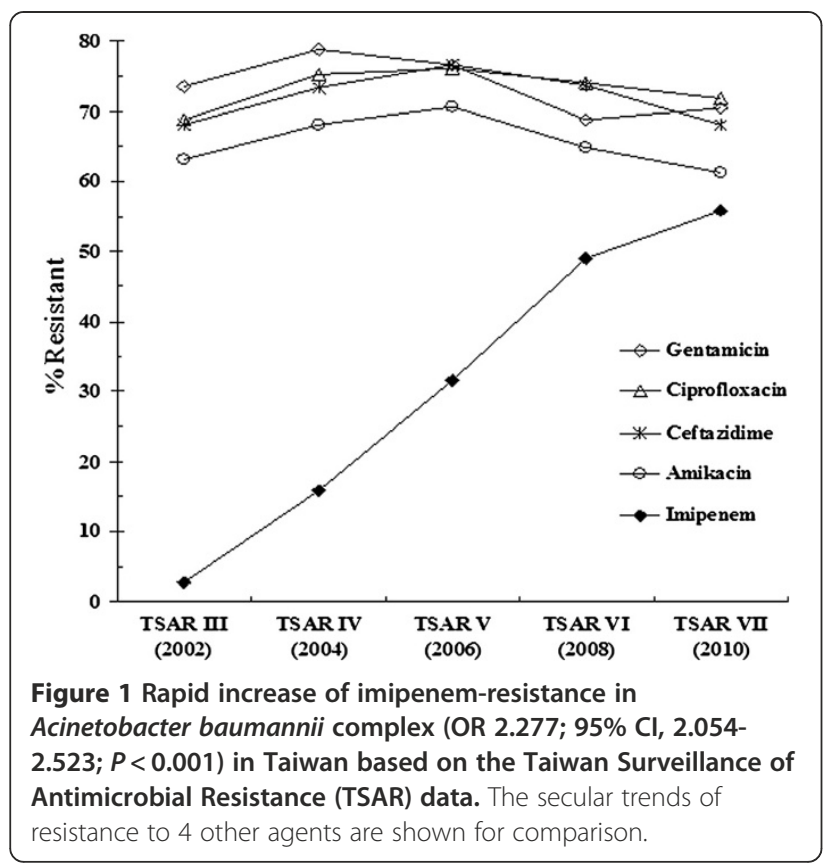

The independent factors associated with XDRABC included recovery from elderly patients, origins other than blood, ICU settings, geographic region, and latter collection year (Table 4).

\section{Discussion}

This nationwide longitudinal study of $1,640 \mathrm{ABC}$ over 10 years revealed the continuous increase of nonsusceptibility to imipenem and emergence of XDRABC. For most other antibiotics, resistance rate increased from 2002 to 2006 but ceased to increase or even decreased thereafter. The prevalence of IRABC and $\mathrm{XDRABC}$ was independently affected by age of patients, specimen types, healthcare settings, and geographic regions.

Resistance to imipenem, which is often accompanied with resistance to multiple other agents, has increased in all parts of the world, ranging from $14.1 \%$ in Europe to $39.4 \%$ in Latin America in 2004-2006 [24], and from $34.5 \%$ in 2006 to $59.8 \%$ in 2009 overall worldwide [9]. Our study revealed the rapid increase in the prevalence of imipenem-resistance over 10 years in Taiwan, from $3.4 \%$ in 2002 to $58.7 \%$ in 2010. The imipenemnonsusceptible rate of $62.6 \%$ in isolates from AsiaPacific in 2009 reported by the SENTRY study was comparable to our result [9].

Although XDRABC in Taiwan has been observed in other pilot studies [10,14], this is the first study addressing the emergence of XDRABC in Taiwan over a long period. However, the variety of definition regarding XDR precluded the comparison of our data with previous
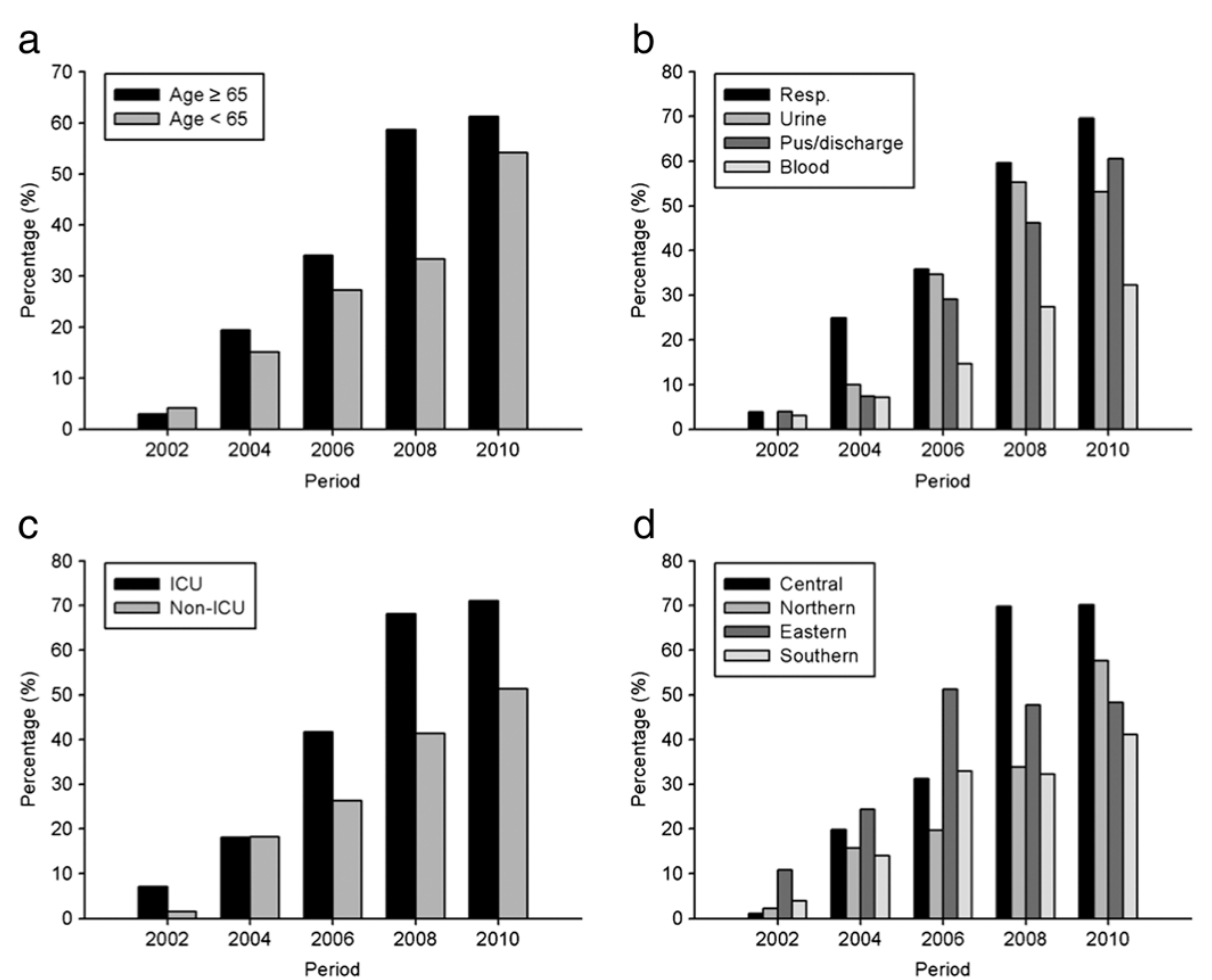

Figure 2 Secular trend of imipenem-resistance rate in Acinetobacter baumannii complex recovered from 2002 to 2010 in Taiwan. Differences in resistance rates were stratified by (a) patient age, (b) sample origins, (c) healthcare settings, and (d) geographic regions, based on the Taiwan Surveillance of Antimicrobial Resistance (TSAR) data. 
Table 3 Factors associated with imipenem-resistant Acinetobacter baumannii complex (IRABC) in Taiwan

\begin{tabular}{|c|c|c|c|c|c|c|}
\hline Characteristic & Non-IRABC & IRABC & $P^{\mathrm{a}}$ & $\mathrm{OR}^{\mathbf{b}}$ & $95 \% \mathrm{Cl}^{\mathrm{b}}$ & $P^{\mathrm{b}}$ \\
\hline Number & 1060 & 580 & & & & \\
\hline Mean age $\pm S D^{c}$ & $65.0 \pm 20.2$ & $70.0 \pm 18.8$ & $<0.001$ & & & \\
\hline Age 65 and older ${ }^{c}$ & $629(61.1)$ & $401(71.9)$ & $<0.001$ & 1.464 & $1.122-1.912$ & 0.005 \\
\hline \multicolumn{7}{|l|}{ Specimen types } \\
\hline Respiratory tracts & $481(45.4)$ & $341(58.8)$ & $<0.001$ & 2.597 & $1.888-3.570$ & $<0.001$ \\
\hline Blood & $192(18.1)$ & $52(9.0)$ & $<0.001$ & & & Reference \\
\hline Pus/discharge & $160(15.1)$ & $59(10.2)$ & 0.005 & 1.934 & $1.240-3.018$ & 0.004 \\
\hline Urine & $140(13.2)$ & $76(13.1)$ & $>0.99$ & 2.138 & $1.401-3.263$ & $<0.001$ \\
\hline \multicolumn{7}{|l|}{ Healthcare settings } \\
\hline Medical centers & $507(47.8)$ & $228(39.3)$ & 0.001 & 0.814 & $0.631-1.049$ & 0.112 \\
\hline ICU Settings & $328(30.9)$ & $272(46.9)$ & $<0.001$ & 1.919 & $1.482-2.486$ & $<0.001$ \\
\hline \multicolumn{7}{|l|}{ Geographic regions } \\
\hline Northern & $336(31.7)$ & $136(23.4)$ & $<0.001$ & & & Reference \\
\hline Central & $341(32.2)$ & $286(49.3)$ & $<0.001$ & 2.43 & $1.791-3.296$ & $<0.001$ \\
\hline Southern & $258(24.3)$ & $85(14.7)$ & $<0.001$ & 1.178 & $0.812-1.710$ & 0.388 \\
\hline Eastern & $125(11.8)$ & $73(12.6)$ & 0.635 & 2.24 & $1.489-3.370$ & $<0.001$ \\
\hline TSAR period ${ }^{d}$ & & & & 2.277 & $2.054-2.523$ & $<0.001$ \\
\hline
\end{tabular}

${ }^{\mathrm{a}} P$ value by chi-square test.

${ }^{\mathrm{b}} \mathrm{P}$ value by multivariate analysis; $\mathrm{OR}$, odds ratio; $\mathrm{Cl}$, confidence interval.

'The patient ages for 50 patients were unknown.

dTaiwan Surveillance of Antimicrobial Resistance (TSAR) III (2002) to VII (2010).
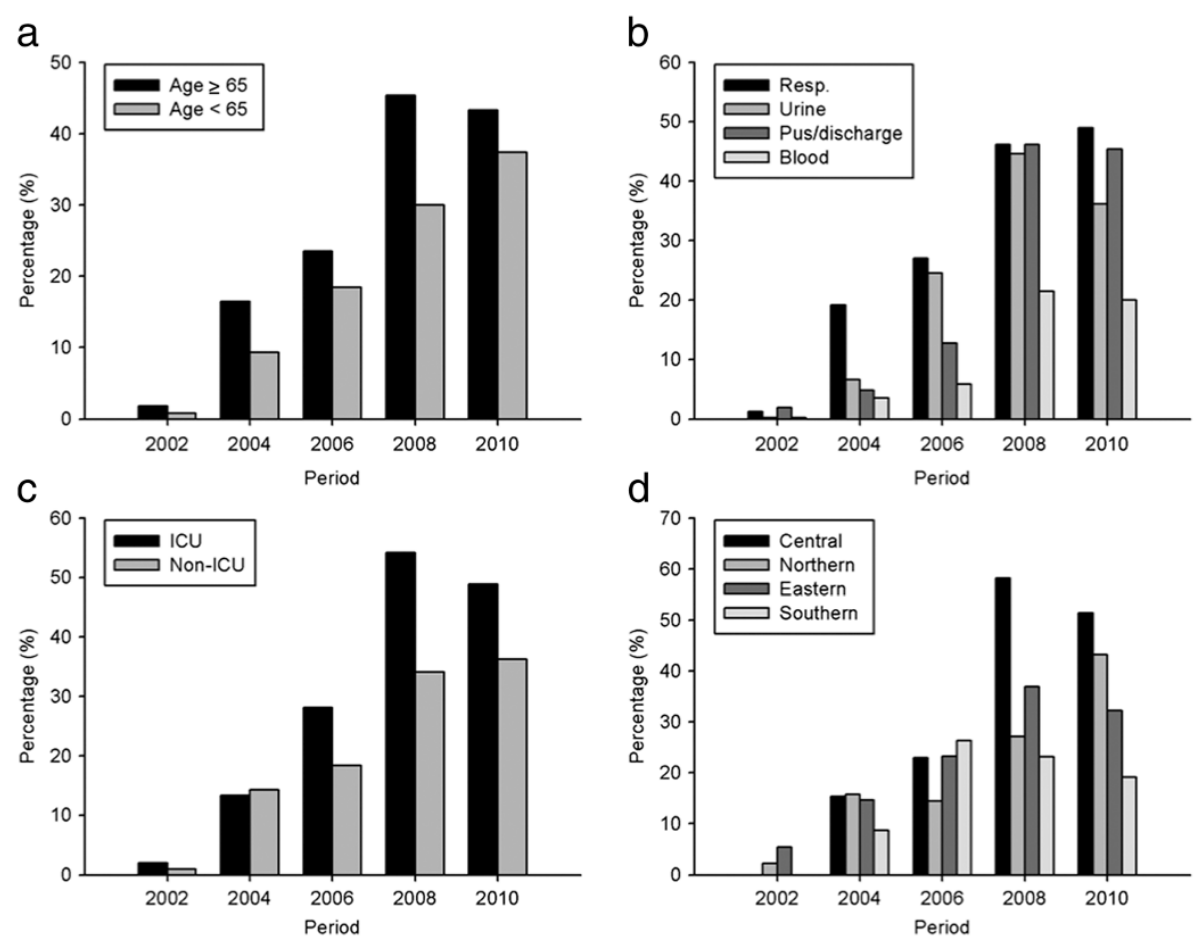

Figure 3 Secular trend of extensively drug-resistance rate in Acinetobacter baumannii complex recovered from 2002 to 2010 in Taiwan. Differences in resistance rates were stratified by (a) patient age (b) sample origins (c) healthcare settings (d) geographic regions based on the Taiwan Surveillance of Antimicrobial Resistance (TSAR) data. 
Table 4 Factors associated with extensively drug-resistant Acinetobacter baumannii complex (XDRABC) in Taiwan

\begin{tabular}{|c|c|c|c|c|c|c|}
\hline Characteristic & Non-XDRABC & XDRABC & $P^{\mathrm{a}}$ & $O R^{b}$ & $95 \% \mathrm{Cl}^{\mathrm{b}}$ & $P^{\mathbf{b}}$ \\
\hline Number & 1212 & 428 & & & & \\
\hline Mean age $\pm S D^{c}$ & $65.4 \pm 20.2$ & $70.6 \pm 18.1$ & $<0.001$ & & & \\
\hline Age 65 and older ${ }^{c}$ & $733(71.2)$ & 297 (28.8) & $<0.001$ & 1.39 & $1.052-1.836$ & 0.021 \\
\hline \multicolumn{7}{|l|}{ Specimens } \\
\hline Respiratory tracts & $571(47.1)$ & $251(58.6)$ & $<0.001$ & 2.102 & $1.507-2.932$ & $<0.001$ \\
\hline Blood & $210(17.3)$ & $34(7.9)$ & $<0.001$ & & & Reference \\
\hline Pus/discharge & $176(14.5)$ & $43(10.0)$ & 0.02 & 1.598 & $0.996-2.568$ & 0.052 \\
\hline Urine & $160(13.2)$ & $56(13.1)$ & $>0.99$ & 1.759 & $1.130-2.739$ & 0.012 \\
\hline \multicolumn{7}{|l|}{ Healthcare settings } \\
\hline Medical centers & $570(47.0)$ & 165 (38.6) & 0.003 & 0.804 & $0.617-1.046$ & 0.104 \\
\hline ICU settings & $404(33.3)$ & $196(45.8)$ & $<0.001$ & 1.468 & $1.126-1.915$ & 0.005 \\
\hline \multicolumn{7}{|l|}{ Geographic regions } \\
\hline Northern & $365(30.1)$ & $107(25.0)$ & 0.047 & & & Reference \\
\hline Central & $404(33.3)$ & $223(52.1)$ & $<0.001$ & 1.965 & $1.466-2.671$ & $<0.001$ \\
\hline Southern & $290(23.9)$ & $53(12.4)$ & $<0.001$ & 0.814 & $0.544-1.216$ & 0.314 \\
\hline Eastern & $153(12.6)$ & $45(10.5)$ & 0.263 & 1.303 & $0.847-2.006$ & 0.229 \\
\hline TSAR period ${ }^{d}$ & & & & 1.97 & $1.773-2.189$ & $<0.001$ \\
\hline
\end{tabular}

${ }^{\mathrm{a}} P$ value by chi-square test.

${ }^{\mathrm{b}} \mathrm{P}$ value by multivariate analysis; $\mathrm{OR}$, odds ratio; $\mathrm{Cl}$, confidence interval.

'The patient ages of 50 patients were unknown.

${ }^{\mathrm{d}}$ Taiwan Surveillance of Antimicrobial Resistance (TSAR) III (2002) to VII (2010).

ones. The definition of XDR in our study was in accordance to that of Tan et al. [21] and approached the consensus of European Centre for Disease Prevention and Control [20] except that not all isolates were tested for trimethoprim-sulfamethoxazole. However, almost all of XDRABC tested showed resistance to it.

Prolonged hospitalization, ICU stay, invasive medical procedures, and prior broad-spectrum antibiotic use, especially carbapenem, third-generation cephalosporins, and fluoroquinolones, have been shown to be risk factors for acquisition of IRABC $[4,7,25]$. These factors, as well as clonal dissemination [26,27], likely all contributed to the increased prevalence of IRABC and XDRABC in Taiwan over the years. A recent study found carbapenem use in Taiwan hospitals increased by $86 \%$ between 2003 and 2008, which was significantly associated with increased healthcare associated IRABC infections [28]. The same study also found that the largest increase in carbapenem use occurred in the central region, the area we found the highest prevalence of IRABC and XDRABC. Strain variability and differences in oxacillinase distribution $[26,27]$ may also account for regional differences and the changes in resistance after 2006, and remain to be investigated.

Based on the independent associated factors we identified, the prevalence of imipenem-resistance and $\mathrm{XDRABC}$ varied greatly. To our knowledge, variations in resistance profiles among $A B C$ from different sample origins have rarely been addressed before. Whether the lower resistance of isolates from blood compared to other sample origins resulted from different clonality and/or impaired virulence in the cost of resistance [29] remained to be elucidated. Varied prevalence among different areas and healthcare settings (ICU) in Asia has been observed [10]. The insignificant difference between medical centers and regional hospitals may have resulted from frequent transportation of patients and high density of hospitals in Taiwan. The advanced age may imply the underlying conditions of the patients. Higher prevalence of IRABC and XDRABC in ICU and regional variations within Taiwan likely reflect differences in patient populations and antibiotic use $[28,30]$.

The rate of resistance to polymyxin B in our study was only $0.2 \%$, which is similar to that found in isolates from the Asia-Western Pacific region between 2006 and 2009 [9]. In contrast, one study in Korea reported a resistance rate of $18.1 \%$ [31]. As for tigecycline, $0.2 \%$ of IRABC isolates had $\mathrm{MIC}>2 \mathrm{mg} / \mathrm{L}$ in Asia region [32] whereas our study found it to be $1.9 \%$ in Taiwan. Susceptibility to polymyxin remained high even for the XDRABC isolates in our study. However, in a pilot study by Jean et al., [14], the authors reported higher rate of resistance to colistin and tigecycline in Taiwan. The source of isolates differed in the two studies. Ours were from different patient groups of both teaching and regional hospitals while theirs were from ICUs of ten 
major teaching hospitals. The underlying conditions, disease severity, and amount of colistin and tigecycline used would be different. However, both studies revealed that compared to other antibiotics, tigecycline and polymyxin B (or its less toxic derivatives, colistin) remained an in vitro effective antimicrobial agent for the treatment of IRABC or XDRABC in Taiwan.

\section{Conclusion}

This longitudinal multicenter surveillance program revealed significant increase and nationwide emergence of IRABC and XDRABC in Taiwan despite that nonsusceptibility to other antibiotics remained stable or declined over the past 6 years. This study also identified factors associated with their resistance to help guide empirical therapy and at-risk groups requiring more intense interventional infection control measures with focused surveillance efforts.

\section{Abbreviations}

IRABC, Imipenem-Resistant Acinetobacter baumannii Complex: XDRABC, Extensively Drug-Resistant Acinetobacter baumannii Complex.

\section{Competing interests}

The authors declare that they have no competing interests.

\section{Authors' contributions}

SCK performed data analysis and interpretation, and drafted the manuscript SCC participated in the design and data interpretation of the study and helped to finalize the manuscript.

HYW, JFL, PCC, YRS and IWH carried out the laboratory assays and participated in data analysis.

TLL designed and supervised the study, participated in data analysis and interpretation, and finalized the manuscript.

All authors read and approved the final version of the manuscript.

\section{Acknowledgements}

We thank the following 26 hospitals for participating in the Taiwan Surveillance of Antimicrobial Resistance (TSAR): Buddhist Tzu Chi General Hospital, Cathay General Hospital, Changhua Christian Hospital, Cheng-Ching Hospital, Chung Shan Medical University Hospital, Ditmanson Medical Foundation Chia-Yi Christian Hospital, Far Eastern Memorial Hospital, HuaLien Hospital, Jen-Ai Hospital, Kaohsiung Armed Forces General Hospital, Kaohsiung Chang Gung Memorial Hospital of the C. G. M. F, Kaohsiung Medical University Chung-Ho Memorial Hospital, Kaohsiung Veterans General Hospital, Kuang Tien General Hospital, Lo-Hsu Foundation, Inc., - Lotung PohAi Hospital, Mennonite Christian Hospital, Min-Sheng Healthcare, National Cheng Kung University Hospital, Saint Mary's Hospital Luodong, Show Chwan Memorial Hospital, Tungs' Taichung MetroHarbor Hospital, Taichung Veterans General Hospital, Tainan Sin-Lau Hospital - the Presbyterian Church in Taiwan, Taipei City Hospital Heping Fuyou Branch, Taipei City Hospital Zhongxiao Branch, Tri-Service General Hospital.

We express our gratitude to Kuang-Hung Chen and Mei-Chun Chiang (Institute of Public Health, School of Medicine, National Yang-Ming University) for their help in the statistics. This project was supported by an intramural grant from the National Health Research Institutes. (ID-098-PP-01 and ID-099-PP-01).

\section{Author details}

${ }^{1}$ National Institute of Infectious Diseases and Vaccinology, National Health Research Institutes, No. 35 Keyan Road, Zhunan, Taiwan 35053. ${ }^{2}$ Institute of Clinical Medicine, National Yang-Ming University, School of Medicine, Taipei, Taiwan. ${ }^{3}$ Division of Infectious Diseases, Department of Medicine, Taipei Veterans General Hospital, Taipei, Taiwan. ${ }^{4}$ Division of Infectious Diseases, Department of Internal Medicine, National Taiwan University Hospital, Taipei,
Taiwan. ${ }^{5}$ Graduate Institute of Clinical Pharmacy, College of Medicine, National Taiwan University, Taipei, Taiwan.

Received: 11 June 2012 Accepted: 23 August 2012

Published: 28 August 2012

\section{References}

1. Peleg AY, Seifert H, Paterson DL: Acinetobacter baumannii: emergence of a successful pathogen. Clin Microbiol Rev 2008, 21:538-582.

2. Dijkshoorn L, Nemec A, Seifert $H$ : An increasing threat in hospitals: multidrug-resistant Acinetobacter baumannii. Nat Rev Microbiol 2007, 5:939-951.

3. Neonakis IK, Spandidos DA, Petinaki E: Confronting multidrug-resistant Acinetobacter baumannii: a review. Int J Antimicrob Agents 2011, 37:102-109.

4. Perez F, Hujer AM, Hujer KM, Decker BK, Rather PN, Bonomo RA: Global challenge of multidrug-resistant Acinetobacter baumannii. Antimicrob Agents Chemother 2007, 51:3471-3484.

5. Kwon KT, Oh WS, Song JH, Chang HH, Jung SI, Kim SW, Ryu SY, Heo ST, Jung DS, Rhee JY, et al: Impact of imipenem resistance on mortality in patients with Acinetobacter bacteraemia. J Antimicrob Chemother 2007, 59:525-530.

6. Sunenshine RH, Wright M-O, Maragakis LL, Harris AD, Song X, Hebden J, Cosgrove SE, Anderson A, Carnell J, Jernigan DB, et al: Multidrug-resistant Acinetobacter infection mortality rate and length of hospitalization. Emerg Infect Dis 2007, 13:97-103.

7. Peleg AY, Paterson DL: Multidrug-resistant Acinetobacter: a threat to the antibiotic era. Intern Med J 2006, 36:479-482.

8. Turner PJ: Meropenem activity against European isolates: report on the MYSTIC (Meropenem Yearly Susceptibility Test Information Collection) 2006 results. Diagn Microbiol Infect Dis 2008, 60:185-192.

9. Gales AC, Jones RN, Sader HS: Contemporary activity of colistin and polymyxin B against a worldwide collection of Gram-negative pathogens: results from the SENTRY Antimicrobial Surveillance Program (2006-09). J Antimicrob Chemother 2011, 66:2070-2074.

10. Jean S-S, Hsueh P-R: High burden of antimicrobial resistance in Asia. Int J Antimicrob Agents 2011, 37:291-295.

11. Lauderdale TL, Clifford McDonald L, Shiau YR, Chen PC, Wang HY, Lai JF, Ho M: The status of antimicrobial resistance in Taiwan among gramnegative pathogens: the Taiwan surveillance of antimicrobial resistance (TSAR) program, 2000. Diagn Microbiol Infect Dis 2004, 48:211-219.

12. White AR, Surveillance BWPoR: The British Society for Antimicrobial Chemotherapy Resistance Surveillance Project: a successful collaborative model. J Antimicrob Chemother 2008, 62(2):ii3-ii14.

13. Tseng SH, Lee CM, Lin TY, Chang SC, Chang FY: Emergence and spread of multi-drug resistant organisms: think globally and act locally. J Microbiol Immunol Infect 2011, 44:157-165.

14. Jean S-S, Hsueh P-R, Lee W-S, Chang H-T, Chou M-Y, Chen I-S, Wang J-H, Lin C-F, Shyr J-M, Ko W-C, et al: Nationwide surveillance of antimicrobial resistance among non-fermentative Gram-negative bacteria in Intensive Care Units in Taiwan: SMART programme data 2005. Int J Antimicrob Agents 2009, 33:266-271

15. Ho M, McDonald LC, Lauderdale TL, Yeh LL, Chen PC, Shiau YR: Surveillance of antibiotic resistance in Taiwan, 1998. J Microbiol Immunol Infect 1999, 32:239-249

16. Chen FJ, Huang IW, Wang CH, Chen PC, Wang HY, Lai JF, Shiau YR, Lauderdale TL: mecA-positive Staphylococcus aureus with low-level oxacillin MIC in Taiwan. J Clin Microbiol 2012, 50:1679-1683.

17. Wu H, Wang JT, Shiau YR, Wang HY, Lauderdale TL, Chang SC: A multicenter surveillance of antimicrobial resistance on Stenotrophomonas maltophilia in Taiwan. J Microbiol Immunol Infect 2012, 45:120-126.

18. Schreckenberger PC, von Graevenitz A: Acinetobacter, Achromobacter, Alcaligenes, Moraxella, and other nonfermentative Gram-negative rods. p. 539-560. In Manual of Clinical Microbiology. 7th edition. Edited by Murray PR, Baron EJ, Pfaller MA, Tenover FC, Yolken RH. Washington, D.C.: American Society for Microbiology; 1999.

19. Clinical and Laboratory Standards Institute (CLSI): Performance Standards for Antimicrobial Susceptibility testing; Twenty-First Information Supplement. CLSI, Wayne, PA: CLSI document M100-S21; 2011. 
20. Magiorakos AP, Srinivasan A, Carey RB, Carmeli Y, Falagas ME, Giske CG, Harbarth S, Hindler JF, Kahlmeter G, Olsson-Liljequist B, et al: Multidrugresistant, extensively drug-resistant and pandrug-resistant bacteria: an international expert proposal for interim standard definitions for acquired resistance. Clin Microbiol Infect 2012, 18:268-281.

21. Tan TY, Lim TP, Lee WH, Sasikala S, Hsu LY, Kwa AL: In vitro antibiotic synergy in extensively drug-resistant Acinetobacter baumannii: the effect of testing by time-kill, checkerboard, and Etest methods. Antimicrob Agents Chemother 2011, 55:436-438.

22. Stelling JM, O'Brien TF: Surveillance of antimicrobial resistance: the WHONET program. Clin Infect Dis 1997, 24(Suppl 1):S157-168.

23. Scarmeas N, Luchsinger JA, Schupf N, Brickman AM, Cosentino S, Tang MX Stern Y: Physical activity, diet, and risk of Alzheimer disease. JAMA 2009, 302:627-637.

24. Reinert RR, Low DE, Rossi F, Zhang X, Wattal C, Dowzicky MJ: Antimicrobial susceptibility among organisms from the Asia/Pacific Rim, Europe and Latin and North America collected as part of TEST and the in vitro activity of tigecycline. J Antimicrob Chemother 2007, 60:1018-1029.

25. Falagas ME, Kopterides P: Risk factors for the isolation of multi-drugresistant Acinetobacter baumannii and Pseudomonas aeruginosa: a systematic review of the literature. J Hosp Infect 2006, 64:7-15.

26. Lin MF, Kuo HY, Yeh HW, Yang CM, Sung CH, Tu CC, Huang ML, Liou ML: Emergence and dissemination of bla(OXA-23)-carrying imipenemresistant Acinetobacter sp. in a regional hospital in Taiwan. J Microbiol Immunol Infect 2011, 44:39-44.

27. Lu PL, Doumith M, Livermore DM, Chen TP, Woodford N: Diversity of carbapenem resistance mechanisms in Acinetobacter baumannii from a Taiwan hospital: spread of plasmid-borne OXA-72 carbapenemase. J Antimicrob Chemother 2009, 63:641-647.

28. Su CH, Wang JT, Hsiung CA, Chien L, Chi CL, Yu HT, Chang FY, Chang SC Increase of carbapenem-resistant Acinetobacter baumannii infection in acute care hospitals in Taiwan: association with hospital antimicrobial usage. Plos One 2012, 7:e37788.

29. Smani Y, Lopez-Rojas R, Dominguez-Herrera J, Docobo-Perez F, Marti S, Vila J, Pachon J: In vitro and in vivo reduced fitness and virulence in ciprofloxacin-resistant Acinetobacter baumannii. Clin Microbiol Infect 2012, 18:E1-E4.

30. Cao J, Song W, Gu B, Mei YN, Tang JP, Meng L, Yang CQ, Wang H, Zhou H: Correlation between carbapenem consumption and antimicrobial resistance rates of Acinetobacter baumannii in a university-affiliated hospital in China. J Clin Pharmacol 2012, Feb 02 [Epub ahead of print].

31. Ko KS, Suh JY, Kwon KT, Jung S-I, Park K-H, Kang Cl, Chung DR, Peck KR, Song $\mathrm{J}-\mathrm{H}$ : High rates of resistance to colistin and polymyxin $\mathrm{B}$ in subgroups of Acinetobacter baumannii isolates from Korea. J Antimicrob Chemother 2007, 60:1163-1167.

32. Farrell DJ, Turnidge JD, Bell J, Sader HS, Jones RN: The in vitro evaluation of tigecycline tested against pathogens isolated in eight countries in the Asia-Western Pacific region (2008). J Infect 2010, 60:440-451.

doi:10.1186/1471-2334-12-200

Cite this article as: Kuo et al.: Emergence of extensively drug-resistant Acinetobacter baumannii complex over 10 years: Nationwide data from the Taiwan Surveillance of Antimicrobial Resistance (TSAR) program. BMC Infectious Diseases 2012 12:200.

\section{Submit your next manuscript to BioMed Central and take full advantage of:}

- Convenient online submission

- Thorough peer review

- No space constraints or color figure charges

- Immediate publication on acceptance

- Inclusion in PubMed, CAS, Scopus and Google Scholar

- Research which is freely available for redistribution

Submit your manuscript at www.biomedcentral.com/submit 